\title{
Derangement of Ras-Guanine Nucleotide-Releasing Factor 1 (Ras-GRF1) and Extracellular Signal-Regulated Kinase (ERK) Dependent Striatal Plasticity in L-DOPA-Induced Dyskinesia
}

\author{
Milica Cerovic, Vincenza Bagetta, Valentina Pendolino, Veronica Ghiglieri, Stefania Fasano, \\ Ilaria Morella, Neil Hardingham, Andreas Heuer, Alessandro Papale, Francesca Marchisella, \\ Carmela Giampà, Paolo Calabresi, Barbara Picconi, and Riccardo Brambilla
}

Background: Bidirectional long-term plasticity at the corticostriatal synapse has been proposed as a central cellular mechanism governing dopamine-mediated behavioral adaptations in the basal ganglia system. Balanced activity of medium spiny neurons (MSNs) in the direct and the indirect pathways is essential for normal striatal function. This balance is disrupted in Parkinson's disease and in L-3,4dihydroxyphenylalanine (L-DOPA)-induced dyskinesia (LID), a common motor complication of current pharmacotherapy of Parkinson's disease.

\begin{abstract}
Methods: Electrophysiological recordings were performed in mouse cortico-striatal slice preparation. Synaptic plasticity, such as longterm potentiation (LTP) and depotentiation, was investigated. Specific pharmacological inhibitors or genetic manipulations were used to modulate the Ras-extracellular signal-regulated kinase (Ras-ERK) pathway, a signal transduction cascade implicated in behavioral plasticity, and synaptic activity in different subpopulations of striatal neurons was measured.

Results: We found that the Ras-ERK pathway, is not only essential for long-term potentiation induced with a high frequency stimulation protocol (HFS-LTP) in the dorsal striatum, but also for its reversal, synaptic depotentiation. Ablation of Ras-guanine nucleotide-releasing factor 1 (Ras-GRF1), a neuronal activator of Ras proteins, causes a specific loss of HFS-LTP in the medium spiny neurons in the direct pathway without affecting LTP in the indirect pathway. Analysis of LTP in animals with unilateral 6-hydroxydopamine lesions (6-OHDA) rendered dyskinetic with chronic L-DOPA treatment reveals a complex, Ras-GRF1 and pathway-independent, apparently stochastic involvement of ERK.
\end{abstract}

Conclusions: These data not only demonstrate a central role for Ras-ERK signaling in striatal LTP, depotentiation, and LTP restored after L-DOPA treatment but also disclose multifaceted synaptic adaptations occurring in response to dopaminergic denervation and pulsatile administration of L-DOPA.

Key Words: Depotentiation, L-DOPA-induced dyskinesia, long-term potentiation (LTP), long-term depression (LTD), Ras-ERK signaling, Ras-GRF1, striatum

\footnotetext{
C hanges in the synaptic efficacy at corticostriatal synapses are believed to be central not only to the regulation of normal cellular functions of the basal ganglia system but also in pathological conditions such as Parkinson's disease and motor complications associated with prolonged dopamine (DA) replacement pharmacotherapy using L-3,4-dihydroxyphenylalanine (L-DOPA) (L-DOPA-induced dyskinesia [LID]) (1-3). These behavioral alterations, most frequently studied in the dorsal striatum, have been correlated to altered bidirectional synaptic

From the School of Biosciences ( $\mathrm{MC}, \mathrm{NH}, \mathrm{AH})$, Cardiff University, Cardiff, United Kingdom; Fondazione Santa Lucia (VB, VP, VG, CG, PC, BP), IRCCS, Rome; Institute of Experimental Neurology (INSPE) (MC, SF, IM, AP, FM, RB), Division of Neuroscience, IRCCS San Raffaele Scientific Institute, Milano; and Clinica Neurologica (PC), University of Perugia, Ospedale S. Maria della Misericordia, Perugia, Italy.

Address correspondence to Riccardo Brambilla, Ph.D., Institute of Experimental Neurology (INSPE), Division of Neuroscience, IRCCS San Raffaele Scientific Institute, Via Olgettina 58, Milano 20132, Italy; E-mail: brambilla.riccardo@hsr.it.
}

Received Jun 25, 2013; revised Mar 17, 2014; accepted Apr 1, 2014. plasticity-long-term potentiation (LTP), long-term depression (LTD), and depotentiation. Bidirectional synaptic plasticity (LTP and LTD) can be induced in most medium spiny neurons (MSNs), the largest neuronal population of the striatum, by means of high-frequency stimulation (HFS) $(100 \mathrm{~Hz})$ of excitatory afferents $(1,4-6)$.

The MSNs are believed to consist of two distinct populations, the striatonigral (direct) pathway neurons and the striatopallidal (indirect) pathway cells, which predominantly express DA $D_{1}$-like and $\mathrm{D}_{2}$-like receptors, respectively. Corticostriatal synaptic plasticity is impaired in MSNs of DA-depleted experimental models, such as the unilateral 6-hydroxydopamine (6-OHDA)-lesion system (6), and is restored by long-term L-DOPA administration (7). More recent evidence suggests that in DA-denervated animals both types of MSNs undergo a reduction in spine density and a corresponding increase in neuronal excitability, a condition partially reverted by L-DOPA treatment (8). Synaptic alterations have been specifically linked to abnormal involuntary movements (AIMs), the rodent correlate of LID. First, striatal synapses from parkinsonian dyskinetic rats fail to depotentiate, a condition normally obtained when low-frequency stimulation (LFS) protocol is applied after the induction of HFS-induced LTP $(7,9)$. Second, HFS-induced LTD cannot be restored in dyskinetic animals unless phosphodiesterase inhibitors are administered (10).

The Ras-extracellular signal-regulated kinase (Ras-ERK) cascade is an evolutionarily conserved signaling cassette implicated in 
several important cellular responses, including memory formation, synaptic plasticity, and behavioral adaptations in the central nervous system as well as in cell proliferation and survival in most cell types (11-13). In the striatum, the Ras-ERK pathway has been shown to control instrumental learning and memory. Additionally, Ras-ERK signaling modulates responses to drugs of abuse and to chronic administration of L-DOPA in experimental models of LID (14-17). To date, the role of Ras-ERK in corticostriatal plasticity has been assessed only preliminarily under some pathological conditions but not in its normal or in DA-depleted state $(18,19)$. In the present study, we show that Ras-ERK signaling is crucial for the induction of HFS-induced LTP and depotentiation at excitatory corticostriatal synapses but that it is not required for HFS-induced LTD. We found that Ras-guanine nucleotide-releasing factor 1 (Ras-GRF1) a brain-specific upstream component of the ERK pathway, modulates HFS-induced LTP selectively in the direct striatal pathway, suggesting that other factors may control ERK signaling in the indirect pathway. In DA-denervated synapses after prolonged L-DOPA treatment, LTP requires ERK signaling in a complex, Ras-GRF1-independent and pathway-independent manner, in contrast to HFS-LTP in the physiological state. Finally, Ras-GRF1 and ERK signaling in synapses from dyskinetic mice impedes rather than facilitates depotentiation of LTP restored by L-DOPA treatment, confirming a derangement of ERK-mediated mechanisms in this neurophysiological correlate of LID.

\section{Methods and Materials}

A detailed description of all methods and materials can be found in Supplement 1. Generation of Ras-GRF1 knockout (KO) mice has been previously described (20-22). Bac-enhanced green fluorescent protein (EGFP) mice (M4-EGFP and A2A-EGFP) were originally generated by the Gene Expression Nervous System Atlas program at Rockefeller University (www.gensat.org) (23). For lesion and LID studies, mice were injected with 6-OHDA into the right medial forebrain bundle, and AIMs were induced as previously described (22). Brain slices and solutions for electrophysiology were prepared as described elsewhere $(7,24)$. Briefly, extracellular field recordings of glutamate-driven population spikes and intracellular whole-cell
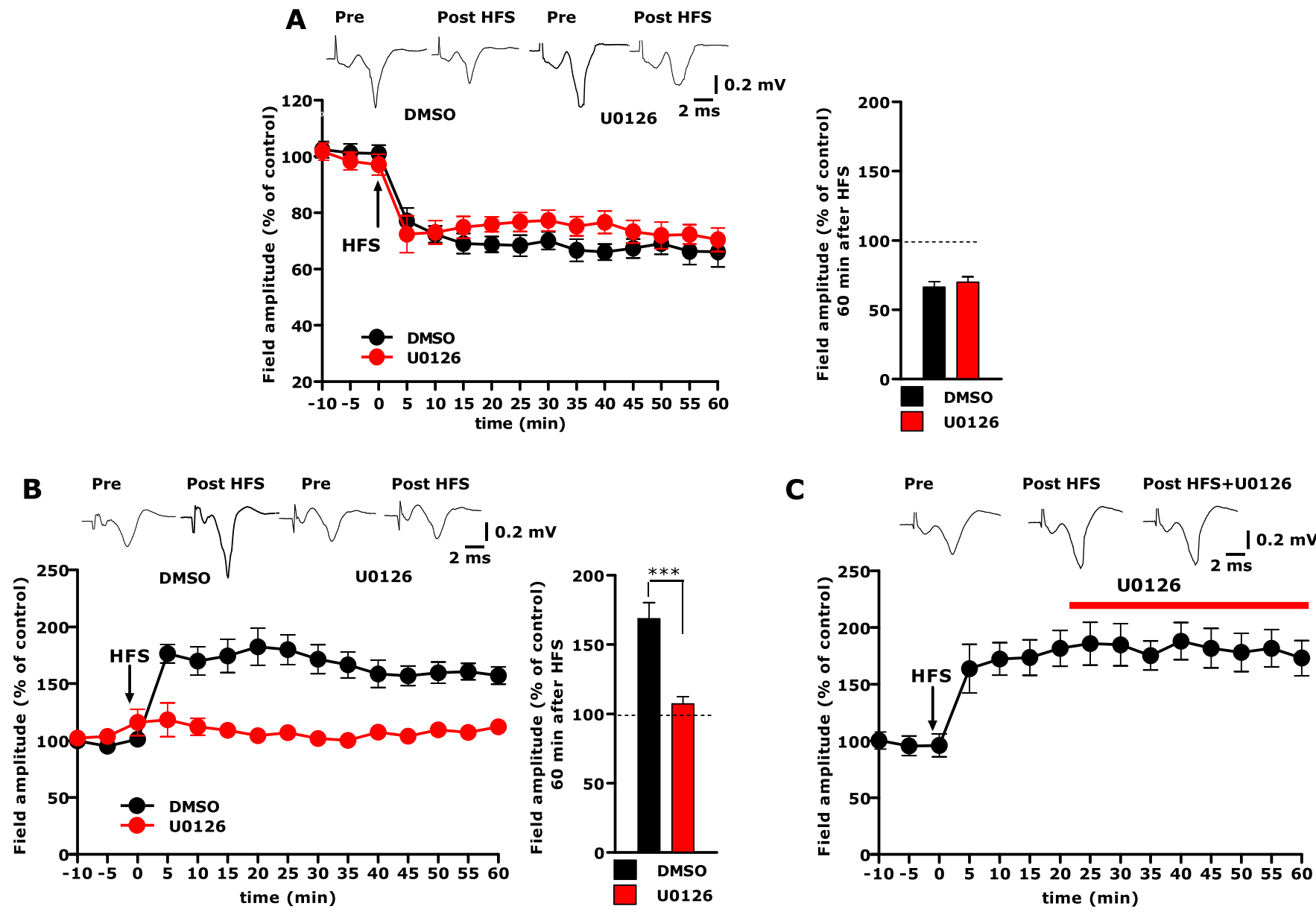

Figure 1. Synaptic responses and long-term plasticity in striatal slices treated with the MEK inhibitor U0126. (A) Left panel shows HFS-LTD was not significantly affected by bathing before incubation (10-20 min) with U0126 (5 $\mu \mathrm{mol} / \mathrm{L})$ (DMSO, $n=6$; U0126, $n=6$; time effect, two-way analysis of variance, $p<.001$ ). Right panel shows field amplitudes as $\%$ of baseline $60 \mathrm{~min}$ after LTD induction were not affected by treatment with U0126 (DMSO $=$ $69.6 \% \pm 2.5 \%$ vs. U0126 $=74.0 \% \pm 3.5 \%$, Student $t$ test, $p>.05$ ). (B) Left panel shows HFS-LTP was blocked by pretreatment with $5 \mu$ mol/L U0126 (DMSO, $n=7 ;$ U0126, $n=7$; treatment effect, two-way analysis of variance, $p<.0001$ ). Right panel shows field amplitudes as \% of baseline 60 min after LTP induction indicate a significant effect of U0126 treatment (DMSO $=166 \% \pm 10.2 \%$ vs. U0126 $=102.6 \% \pm 3.5 \%$, Student $t$ test, ${ }^{* * *} p<.0001$. (C) U0126 $(5 \mu \mathrm{mol} / \mathrm{L})$ does not affect LTP maintenance when applied $20 \mathrm{~min}$ after HFS induction (LTP before U0126 $=178 \% \pm 16.1 \%$ vs. after U0126 $=180.5 \% \pm$ $16.3 \%, n=4$, Student $t$ test, $p>.05$ ). Insets show representative traces before and after HFS in untreated and U0126-treated slices from naïve mice. DMSO, dimethyl sulfoxide; HFS, high-frequency stimulation; LTP, long-term potentiation; MEK, mitogen-activated protein kinase kinase. 
patch recordings were obtained in the dorsolateral striatum where stimuli were delivered via bipolar stimulating electrodes.

Induction of LTD and LTP was accomplished with HFS consisting of three to four trains at $100 \mathrm{~Hz}$ of $1-3 \mathrm{sec}$ duration with $20-\mathrm{sec}$ intratrain intervals. This protocol was used in the presence of $1.2 \mathrm{mmol} / \mathrm{L} \mathrm{Mg}^{2+}$ in recording solution to obtain LTD; LTP was induced in $\mathrm{Mg}^{2+}$-free solution. To depotentiate previously induced LTP, LFS for $10 \mathrm{~min}$ at $2 \mathrm{~Hz}$ was delivered. For immunofluorescence and immunohistochemistry studies, slices were first stimulated with HFS to induce LTP and 10 min later were fixed with $4 \%$ paraformaldehyde. Control slices were perfused 20-30 min with $\mathrm{Mg}^{2+}$-free solution before fixing. These slices were resliced into thinner sections and processed for immunohistochemistry and immunofluorescence according to published procedures $(22,25)$.

\section{Results}

\section{ERK Signaling Is Necessary for HFS-LTP at Corticostriatal Synapse}

The Ras-ERK signaling pathway in the dorsal portion of the striatum is abnormally activated in response to both drugs of abuse and L-DOPA $(15,26)$. Hyperactivation of this signaling cascade may represent a key pathological factor for both drug addiction and LID, two conditions relying on abnormal DAmediated adaptations in the basal ganglia system. In particular, we showed more recently that Ras-GRF1, a neuronal specific guanosine diphosphate/guanosine triphosphate (GDP/GTP) Rasexchange factor, plays a crucial role in the regulation of ERKmediated cellular and behavioral responses to psychostimulants by sensing and integrating DA and glutamate signaling in the striatum. In addition, Ras-GRF1 inhibition, both in murine and in nonhuman primate models of LID, significantly improves the behavioral symptoms linked to repeated L-DOPA administration $(21,22)$. To identify the synaptic correlates of the observed behavioral responses in MSNs, we investigated the role of RasGRF1 and ERK signaling in long-term plasticity at the corticostriatal synapse.

We performed extracellular field recordings of population spike responses in slices obtained from wild-type (WT) mice and exposed to the mitogen-activated protein kinase kinase (MEK) inhibitor U0126 (5 $\mathrm{mol} / \mathrm{L})$, which blocks ERK signaling. This treatment did not affect basal synaptic responses that were consistent within a whole range of stimulus intensities (Figure S1A in Supplement 1). When HFS was delivered in physiological $\mathrm{Mg}^{2+}$ concentration, robust LTD was induced in both vehicle and U0126-treated slices (Figure 1A). Next, we examined corticostriatal LTP. To this purpose, $\mathrm{Mg}^{2+}$ was removed from the extracellular medium, promoting stronger activation of glutamate $\mathrm{N}$-methyl-Daspartate receptors $(1,4-6)$. Under these experimental conditions, HFS-LTP was completely abolished by pretreatment with $5 \mu \mathrm{mol} / \mathrm{L}$ U0126 (Figure 1B, left panel). Analysis of the posttetanic mean amplitudes $(60 \mathrm{~min})$ further confirmed the effect of U0126 treatment (Figure 1B, right panel). We obtained similar results using intracellular recordings where LTP induction was abolished by U0126 treatment in all recorded cells (Figure S1B in Supplement 1).

To test whether ERK is implicated in both LTP maintenance and depotentiation, U0126 was applied 20 min after tetanus in field recordings. This treatment did not affect LTP (Figure 1C). However, pretreatment of potentiated synapses with this MEK inhibitor did prevent depotentiation after LFS (Figure 2).
Altogether, these data indicate that ERK signaling is necessary not only for the induction of HFS-LTP in the dorsal striatum but also for synaptic depotentiation. However, ERK is not required for LTP maintenance, HFS-LTD and basal synaptic transmission.

\section{Ras-GRF1 Controls HFS-LTP in Striatonigral Pathway}

Our data suggest a specific role for ERK signaling in the induction of HFS-LTP but do not implicate Ras-GRF1 in striatal synaptic plasticity. To address this issue, we performed electrophysiological recordings using intracellular sharp electrodes in corticostriatal slices from WT and Ras-GRF1 KO mice (4,5,20). A normal HFS-LTD could be induced in both WT and mutant mice (Figure 3A, left panel). Analysis of the 40-min posttetanic mean amplitudes confirms the lack of genotype effect (Figure 3A, right panel). However, although HFS-LTP could be reliably induced in WT mice, this form of plasticity was present in $\sim 50 \%$ of the striatal MSNs recorded from Ras-GRF1 KO mice (Figure 3B, left panel). Comparison of the 40-min posttetanic mean amplitudes confirms that only one subpopulation of Ras-GRF1 KO MSNs lacked LTP (Figure 3B, right panel). These data indicate that ERK signaling in the dorsal striatum is necessary for HFS-LTP but not

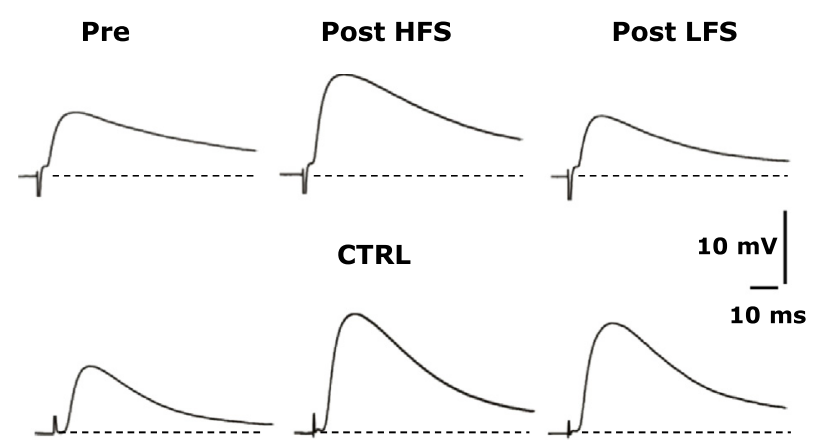

CTRL + U0126

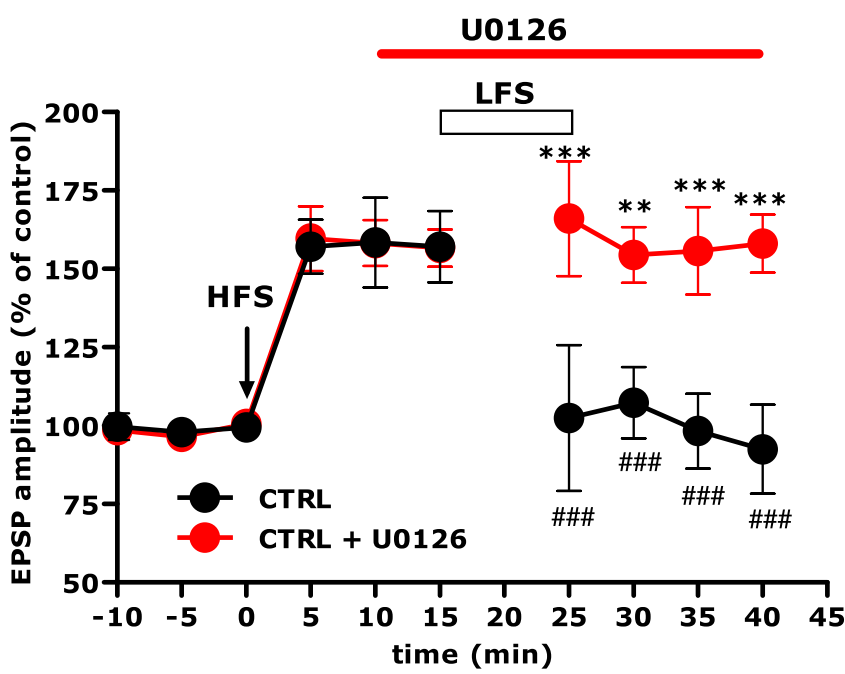

Figure 2. Depotentiation is prevented by application of U0126. LFS-induced depotentiation was blocked by application of $5 \mu \mathrm{mol} / \mathrm{L}$ U0126 (U0126, before vs. after LFS, Student $t$ test, $p>.05, n=6$; CTRL, before vs. after LFS, Student $t$ test, ${ }^{\# \#} p<.0001, n=6$; CTRL vs. U0126, two-way analysis of variance, $p<.0001$; post hoc Bonferroni, ${ }^{* *} p<.001,{ }^{* * *} p<.0001$ ). Inset shows representative traces before HFS, after HFS, and on LFS in naïve and U0126-treated slices. CTRL, control; EPSP, excitatory postsynaptic potential; HFS, highfrequency stimulation; LFS, low-frequency stimulation. 

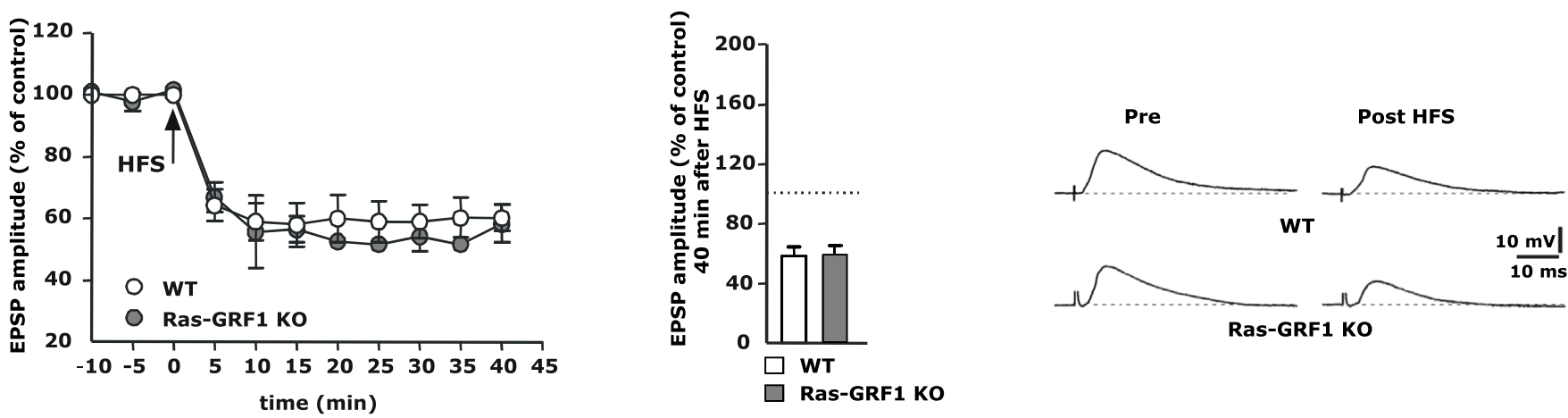

B
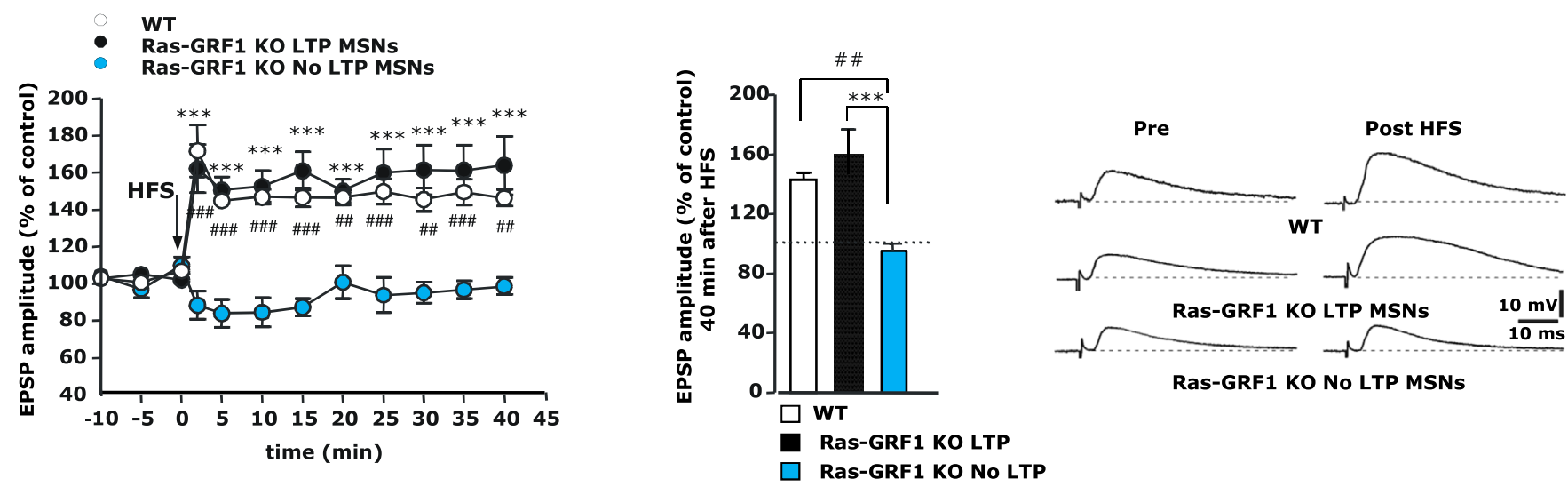

Figure 3. Electrophysiological analysis of the corticostriatal synaptic plasticity recorded from WT and Ras-GRF1 KO mice. (A) Left panel shows HFS protocol induced a normal long-term depression in both WT and mutant mice (WT, $n=8$; Ras-GRF1 KO, $n=9$; time effect, two-way analysis of variance, $p<.001$ ). Bar diagram in the right panel shows the EPSP amplitude as \% of control, measured at 40 min after tetanus, both in WT and in mutant mice (WT $=60.3 \% \pm$ $4.3 \%$ vs. Ras-GRF1 KO $=58.3 \% \pm 6.1 \%$, Student $t$ test, $p>.05$ ). (B) Left panel shows LTP was induced only in half of the striatal MSNs recorded from RasGRF1 KO mice (Ras-GRF1 KO LTP MSNs, $n=8$ vs. Ras-GRF1 KO No LTP MSNs, $n=9$, two-way analysis of variance, $p<.001$; post hoc Bonferroni, *** $p<.001$; WT, $n=5$ vs. Ras-GRF1 KO No LTP MSNs, post hoc Bonferroni, \#\# $p<.01, \# \#-.001$ ). Bar diagram in the right panel shows the mean EPSP amplitudes as \% of control for all three cell populations (WT $=143.1 \% \pm 4.5 \%$, Ras-GRF1 KO LTP $=160.0 \% \pm 15.8 \%$, Ras-GRF1 KO No LTP $=95.2 \% \pm 4.6 \%$; WT vs. KO No LTP, one-way analysis of variance, ${ }^{\# \#} p<.01 ;$ KO LTP vs. KO No LTP, one-way analysis of variance, $\left.{ }^{* * *} p<.001\right)$. Sample traces of EPSP amplitudes before and after HFS in WT and Ras-GRF1 KO mice are shown on the right of each panel. EPSP, excitatory postsynaptic potential; HFS, high-frequency stimulation; LTP, long-term potentiation; KO, knockout; MSN, medium spiny neuron; Ras-GRF1, Ras-guanine nucleotide-releasing factor1; WT, wild-type.

for HFS-LTD and that Ras-GRF1 is likely to play a role only in a specific subset of MSNs.

Because we previously established that the Ras-GRF1 is equally expressed in both striatonigral and striatopallidal MSNs, the pathway-specific involvement of Ras-GRF1 in LTP is likely due to its specific engagement by a subclass of these striatal cells (22). To clarify which subpopulation of MSNs is affected by the loss of RasGRF1, we took advantage of two types of bacterial artificial chromosome transgenic mice expressing enhanced green fluorescent protein (EGFP) specifically in striatonigral (M4-EGFP mice) or striatopallidal (A2A-EGFP mice) MSNs (23). To study the role of RasGRF1 in the two different subpopulations of MSNs, we combined these bacterial artificial chromosome transgenic mutants with RasGRF1 KO mice to obtain double mutants (M4-EGFP/Ras-GRF1 KO and A2A-EGFP/Ras-GRF1 KO). We first performed whole-cell patch clamp recordings from M4-EGFP and A2A-EGFP single transgenic mice to ensure that LTP could be reliably induced in EGFP-positive and EGFP-negative cells in both transgenic lines. We were able to induce LTP, with similar amplitude and time course, in both fluorescent and nonfluorescent MSNs from M4-EGFP mice (Figure S2A in Supplement 1). Similarly, A2A-EGFP mice also showed LTP in both cell populations (Figure S2B in Supplement 1). However, when we subsequently performed recordings in slices obtained from M4-EGFP/Ras-GRF1 KO double mutants, LTP could be induced only in indirect pathway MSNs (iMSNs) (Figure 4A). As a further confirmation that Ras-GRF1 is essential for LTP in direct pathway MSNs (dMSNs), we also performed recordings from A2A-EGFP/RasGRF1 KO double mutants (Figure 4B). This finding provides evidence for the selective involvement of Ras-GRF1 in the control of HFS-LTP in the direct striatal pathway. The residual LTP in naïve Ras-GRF1 KO slices was completely blocked by U0126 treatment, confirming that LTP in the indirect pathway is ERK, but not RasGRF1, dependent (Figure S2C in Supplement 1). When the experiments were performed by measuring extracellular field responses, we found that the magnitude of LTP in Ras-GRF1 KO slices was significantly smaller compared with WT slices as a result of the selective contribution of synaptic potentiation in the iMSNs (Figure S2C in Supplement 1). 
A

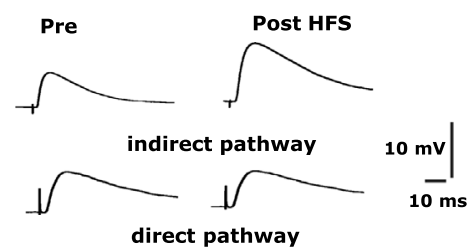

B

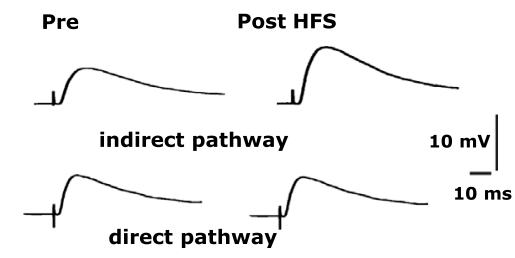

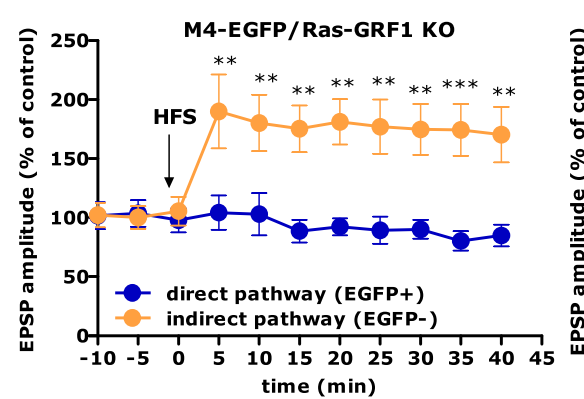
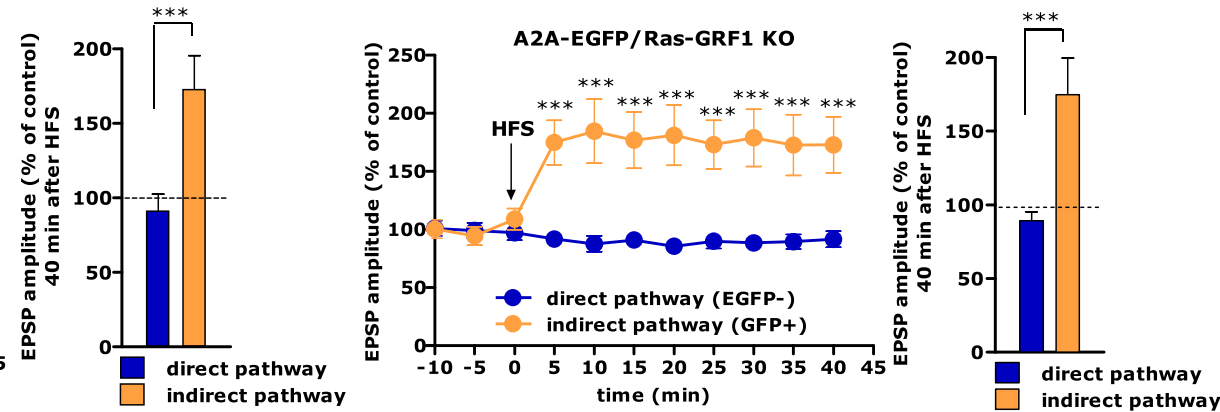

Figure 4. HFS-LTP is absent in the dMSNs from Ras-GRF1 KO mice. (A) Left panel shows in mice expressing EGFP in striatonigral MSNs (M4-EGFP)/RasGRF1 KO mice long-term potentiation was blocked in the direct pathway (dMSNs, $n=5 \mathrm{vs}$. iMSNs, $n=5$, two-way analysis of variance, $p<.001$; post hoc Bonferroni, $\left.{ }^{* *} p<.001,{ }^{* * *} p<.0001\right)$. Right panel shows EPSP amplitudes $40 \mathrm{~min}$ after HFS as $\%$ of baseline (dMSNs $=93.1 \% \pm 8.7 \%$ vs. iMSNs $=173.1 \%$ $\pm 22.3 \%$, Student $t$ test, ${ }^{* * *} p<.0001$ ). (B) Left panel shows HFS-LTP was blocked in the dMSNs from mice expressing EGFP in striatopallidal MSNs (A2AEGFP)/Ras-GRF1 KO mice (dMSNs, $n=6$ vs. iMSNs, $n=4$, two-way analysis of variance, $p<.0001$; post hoc Bonferroni, ${ }^{* * *} p<.0001$ ). Right panel shows EPSP amplitudes $40 \mathrm{~min}$ after HFS as $\%$ of baseline (dMSNs $=89.4 \% \pm 6.1 \%$ vs. iMSNs $=174.7 \% \pm 24.2 \%$, Student $t$ test, ${ }^{* * *} p<.0001$ ). Insets are sample traces of EPSP amplitudes before and after HFS in M4-EGFP/Ras-GRF1 KO and A2A-EGFP/Ras-GRF1 KO mice. dMSN, direct pathway MSN; EGFP, enhanced green fluorescent protein; EPSP, excitatory postsynaptic potential; HFS, high-frequency stimulation; iMSN, indirect pathway MSN; LTP, long-term potentiation; KO, knockout; MSN, medium spiny neuron; Ras-GRF1, Ras-guanine nucleotide-releasing factor1.

To gain direct information about cell signaling processes during LTP induction, we measured the phosphorylation levels of ERK1/2 in acute corticostriatal slices stimulated with the HFS-LTP protocol. The ERK phosphorylation (pERK) levels were significantly higher in stimulated slices compared with control slices, and U0126 treatment fully prevented this increase (Figure S3A in Supplement 1).

To distinguish between downstream effect of ERK in the two striatal pathways, we also measured the phosphorylation levels of ribosomal protein S6 (p-S6) on Ser235/236, a cytoplasmic target of ERK1/2 activity, as well as one of its nuclear targets, histone H3 (p-H3), by using immunofluorescence techniques (25). Similar to pERK1/2, total p-S6 levels were significantly higher in stimulated slices compared with control slices, and this stimulation was shown to be entirely ERK dependent because pretreatment with U0126 completely abolished the signal (Figure S4A in Supplement 1). However, we observed differences in the ability of HFS-LTP to induce S6 phosphorylation in the two pathways. Basal levels of p-S6 appeared to be higher in the indirect pathway compared with the direct pathway, whereas the stimulation effect was similar (Figure S4B,C in Supplement 1). Induction of p-S6 was totally blocked in Ras-GRF1 KO slices but only in the direct pathway (Figure S4B,C in Supplement 1). When $\mathrm{p}-\mathrm{H} 3$ was monitored, we found a similar scenario, with a higher activation in the indirect pathway than in the direct one, with both completely inhibited by U0126 (Figure S5 in Supplement 1). These data confirm that ERK activation is necessary for HFS-LTP in both pathways and that Ras-GRF1 controls ERK1/2 signaling only in the direct pathway, further supporting the observation that in RasGRF1 KO slices only the striatonigral LTP is impaired.

\section{Ras-GRF1 and ERK Signaling Control DA-Mediated Adaptations in the Murine Model of LID}

Parkinson's disease is a neurodegenerative disorder characterized by a slow, inexorable loss of substantia nigra pars compacta dopaminergic neurons and by an ensuing depletion of DA input to the striatum. The most effective treatment for Parkinson's disease is DA replacement therapy, using the DA precursor L-DOPA, but prolonged use of L-DOPA is associated with complications including motor fluctuations and LID, which can limit treatment over time (16,27-29). Considering the central role played by Ras-GRF1 and ERK signaling in HFS-LTP, we assessed the possibility that this intracellular pathway may also be implicated in synaptic alterations in response to both DA depletion and replacement with L-DOPA. We first generated a unilateral 6-OHDA-lesion model of Parkinson's disease by injecting the neurotoxin into the right medial forebrain bundle of both Ras-GRF1 KO mice and control mice (littermates). We measured spontaneous ipsilateral rotations 2 weeks after surgery as an efficacy index of the lesion. The rotational behavior was similar in the two genotypes (Figure S6A in Supplement 1), suggesting an equivalent sensitivity to the neurotoxic damage. To elicit axial, limb, and orolingual AIMs, a validated model of LID in mice, we administered an ascending dose regimen of L-DOPA (3 mg/kg, 6 $\mathrm{mg} / \mathrm{kg}, 12 \mathrm{mg} / \mathrm{kg}$ daily) to both 6-OHDA-lesion WT and Ras-GRF1 mutant mice for 9 consecutive days (22). Daily scoring of AIMs, from day 1-8, revealed gradual development of dyskinetic symptoms in both genotypes, which affected the trunk, limb, and orolingual regions on the side of the body contralateral to the lesion. However, AIMs scores were significantly reduced in Ras-GRF1 KO mice compared with their littermate controls (Figure $\mathrm{S} 6 \mathrm{~B}$ in Supplement 1). These results are consistent with our previous data showing that the absence of Ras-GRF1 strongly attenuates LID induction in mice (22).

After 6-OHDA lesioning, all MSNs recorded from either WT mice or Ras-GRF1 KO mice lost the ability to show LTP, an expected consequence of DA denervation (Figure S6C in Supplement 1) $(4,6)$. L-DOPA treatment has been shown to rescue LTP in DA-denervated rats, but, concomitantly with the 
development of AIMs, depotentiation is occluded (7). We next examined the induction of LTP and its reversal by LFS in slices from Ras-GRF1 KO mice and WT littermates with 6-OHDA lesions and administered long-term L-DOPA treatment. The MSNs intracellularly recorded from the mildly dyskinetic Ras-GRF1 KO mice displayed normal LTP after HFS and a significant depotentiation of the synaptic activity after LFS, suggesting that corticostriatal plasticity parallels the low dyskinetic profile of these mutants. Conversely, although LTP was normal in the highly dyskinetic WT control mice, depotentiation was abolished (Figure 5A).

Although both Ras-GRF1 KO and WT mice showed HFS-LTP in response to chronic L-DOPA administration, LFS (intracellular recording) produced depotentiation only in Ras-GRF1 KO mice, which showed fewer dyskinetic symptoms compared with WT mice. These data clearly indicate that, in contrast to depotentiation in naïve slices, which requires ERK signaling, in dyskinetic slices synaptic depotentiation is facilitated by ERK blockade through RasGRF1 ablation. To support this evidence by a direct pharmacological inhibition of ERK, we treated slices from WT dyskinetic mice with U0126 $10 \mathrm{~min}$ before LFS in field recordings. This treatment restored depotentiation (Figure 5B), confirming that in the dyskinetic condition ERK signaling is part of the mechanism occluding depotentiation, in contrast to the naïve condition, in which ERK instead plays a facilitating role.

The observation that LTP not only was restored after L-DOPA treatment in the Ras-GRF1 mutants in the indirect pathway but also established de novo in the direct pathway indicates that profound cellular adaptations occur in denervated animals subjected to chronic L-DOPA administration. In particular, these data suggest that ERK signaling through Ras-GRF1 may no longer be necessary for LTP induction in L-DOPA-treated mice. To test this hypothesis, we pretreated striatal slices from dyskinetic animals with U0126 (5 $\mu \mathrm{mol} /$ L). Unexpectedly, we detected two distinct but equally represented cell populations: one in which U0126 administration blocked the induction of LTP and the other in which the drug did not have any effect on this form of plasticity (Figure 6A). To exclude the possibility that ERK signaling sensitivity to pharmacologic manipulation was affected by the development of dyskinesia, we repeated the experiment using a higher dose of U0126 $(20 \mu \mathrm{mol} / \mathrm{L})$. Under these experimental conditions, we obtained the same pattern of LTP induction as with the $5-\mu \mathrm{mol} / \mathrm{L}$ dose (Figure $6 \mathrm{~B}$ ).

\section{A}

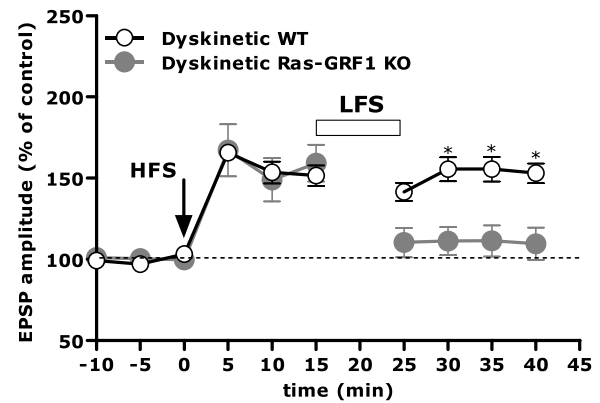

Altogether, this evidence indicates that $\sim 50 \%$ of MSNs can still manifest LTP even in the absence of ERK signaling. One obvious possibility was that the observed segregation in two populations could be related to the two functional pathways, the striatonigral and the striatopallidal. To test this hypothesis, we recorded from EGFP-positive cells obtained from A2A-EGFP and M4-EGFP transgenic dyskinetic mice. As shown in Figure 6C-D, the same distribution pattern was evident, with EGFP-positive cells showing either LTP or no LTP in the presence of U0126 (5 $\mu \mathrm{mol} / \mathrm{L})$, roughly following a 50:50 ratio.

The scenario emerging from our data on L-DOPA-treated animals is complex. On one hand, Ras-GRF1 is not required for LTP in the direct pathway of L-DOPA-treated parkinsonian mice, in contrast to what is observed in the naïve animals, in which loss of Ras-GRF1 completely blocks LTP in the striatonigral MSNs. On the other hand, ERK signaling is still necessary to induce LTP after chronic L-DOPA administration but in a pathway-independent, apparently stochastic manner.

\section{Discussion}

The Ras-ERK signaling pathway is activated in MSNs by a combined engagement of DA and glutamate receptors, making it a suitable candidate for having a regulatory role in striatal synaptic plasticity (14-16). It is now well established that distinct chemical stimuli are able to activate ERK signaling in specific MSN subpopulations. For example, psychostimulants in intact animals and L-DOPA in denervated animals selectively activate the ERK cascade in MSNs of the direct pathway $(30,31)$. The present study provides initial evidence of a complex role of ERK-dependent and Ras-GRF1-dependent signaling in corticostriatal plasticity, highlighting profound differences between synaptic mechanisms in naïve slices and DA-depleted preparations obtained from L-DOPA-treated dyskinetic animals.

First, ERK in naïve slices is vital for LTP induction, but not for its maintenance, in both striatal pathways. Second, the mechanism of ERK activation in response to HFS is distinctly different in the two cell types. In striatonigral MSNs, LTP is Ras-GRF1 dependent, whereas LTP is Ras-GRF1 independent in striatopallidal MSNs. This evidence is unexpected because we previously demonstrated that Ras-GRF1 is expressed in both MSN subpopulations (22).

$\mathbf{B}$

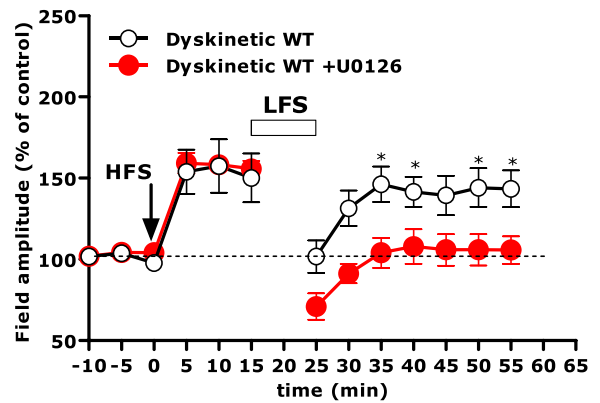

Figure 5. Depotentiation in dyskinetic mice can be restored either by the ablation of Ras-GRF1 or by the specific MEK inhibitor U0126. (A) L-DOPA-treated Ras-GRF1 KO mice showed a normal LTP after HFS. The subsequent LFS protocol induced a significant depotentiation in all MSNs recorded (before LFS vs. after LFS dyskinetic Ras-GRF1 KO, Student $t$ test, $p<.0001$; dyskinetic WT, $n=4$ vs. dyskinetic Ras-GRF1 KO, $n=7$, post hoc Bonferroni, * $p<.05$ ). (B) In slices from dyskinetic WT mice when U0126 (5 $\mu \mathrm{mol} / \mathrm{L})$ was applied $10 \mathrm{~min}$ before LFS, a significant depotentiation was observed (before vs. after LFS dyskinetic WT + U0126, Student $t$ test, $p<.05$; dyskinetic WT, $n=5$ vs. dyskinetic WT + U0126, $n=6$, post hoc Bonferroni, $\left.{ }^{*} p<.05\right)$. EPSP, excitatory postsynaptic potential; HFS, high-frequency stimulation; LTP, long-term potentiation; KO, knockout; MEK, mitogen-activated protein kinase kinase; MSN, medium spiny neuron; Ras-GRF1, Ras-guanine nucleotide-releasing factor1; WT, wild-type. 
A Dyskinetic WT mice<smiles>[C+]C=CC=C</smiles>

LTP MSNs $+5 \mu$ M U0126 - No LTP MSNs $+5 \mu$ M U0126
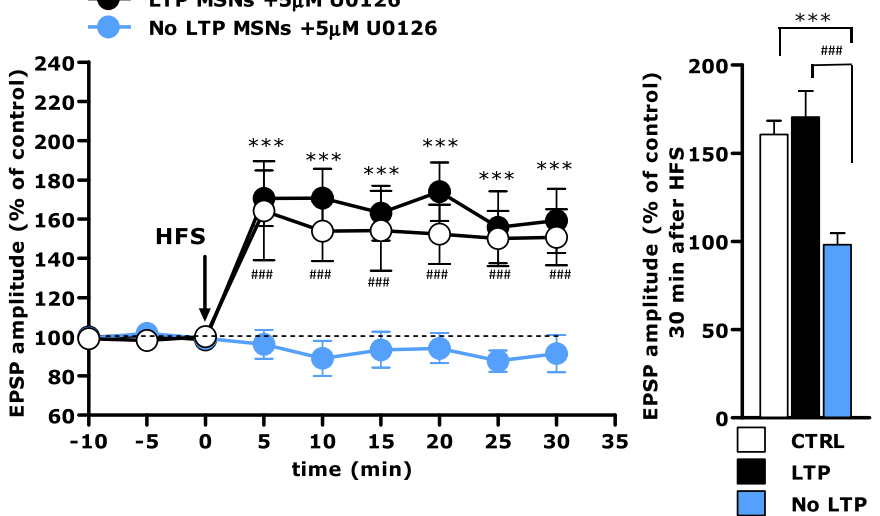

B Dyskinetic WT mice
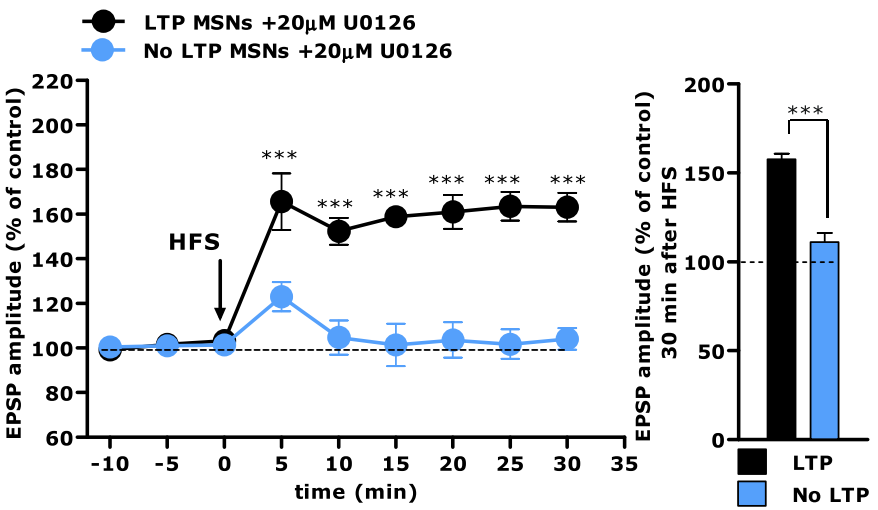

C

Dyskinetic M4-EGFP mice

LTP MSNs (EGFP+)

No LTP MSNS (EGFP+)

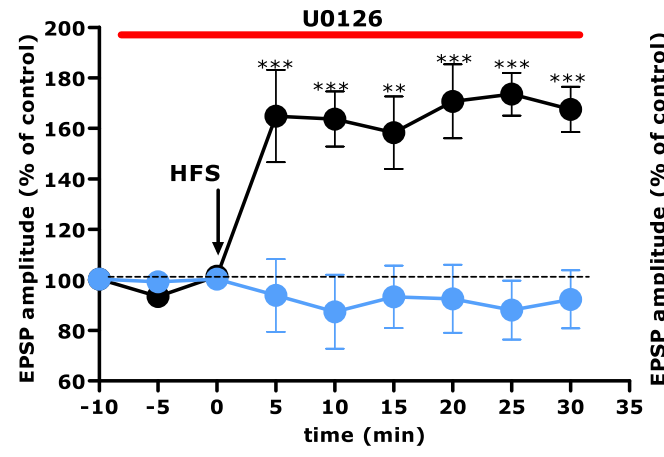

D

Dyskinetic A2A-EGFP mice

LTP MSNs (EGFP+)

No LTP MSNs (EGFP+)

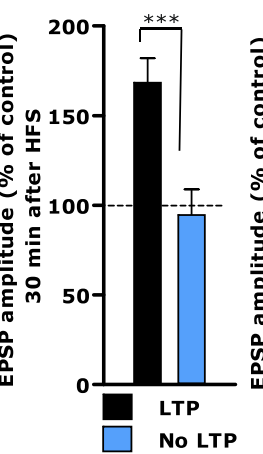

U0126

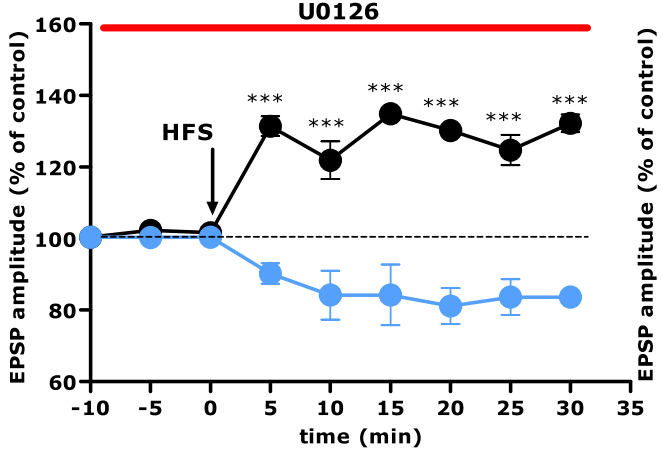

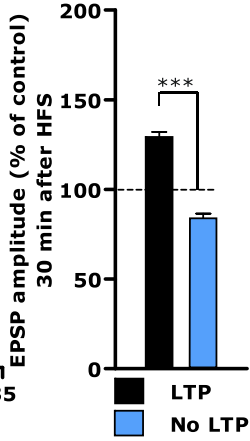

Figure 6. Effects of U0126 on LTP in dyskinetic mice. (A) Left panel shows LTP could be induced in half of MSNs recorded in slices from dyskinetic mice pretreated with U0126 (5 $\mu \mathrm{mol} / \mathrm{L})$ (dyskinetic mice + vehicle, $n=8$ vs. dyskinetic mice + U0126 No LTP MSNs, $n=6$, post hoc Bonferroni, \#\#\# $p<.001$; dyskinetic mice + U0126 LTP MSNs, $n=6$ vs. dyskinetic mice + U0126 No LTP MSNs, $n=6$, post hoc Bonferroni, $\left.{ }^{* * *} p<.001\right)$. Bar diagram in the right panel shows the mean of EPSP amplitude as $\%$ of control for all three cell populations (CTRL $=160 \% \pm 7.9 \%$, No LTP MSNs $=92.2 \% \pm 6.5 \%$, LTP MSNs $=170.1 \% \pm 14.8 \%$, oneway analysis of variance, ${ }^{\# \# \#} p<.001,{ }^{* * *} p<.001$ ). (B) Left panel shows the application of $20 \mu \mathrm{mol} / \mathrm{L}$ U0126 prevented LTP induction in half of the recorded MSNs from dyskinetic WT mice (LTP MSNs, $n=6$ vs. No LTP MSNs, $n=7$, two-way analysis of variance, $p<.001$ ). Histogram in the right panel shows the mean EPSP amplitude as $\%$ of control $30 \mathrm{~min}$ after HFS for both cell populations (No LTP MSNs $=111.0 \% \pm 5.2 \%$ vs. LTP MSNs $=157.0 \% \pm 3.2 \%$, Student $t$ test, ${ }^{* * *} p<.001$ ). (C) Left panel shows in dyskinetic mice expressing EGFP in dMSNs (M4-EGFP) the application of $5 \mu$ mol of U0126 prevented LTP induction in half of the fluorescent dMSNs (LTP M4-EGFP MSNs, $n=4$ vs. No LTP M4-EGFP MSNs, $n=4$, post hoc Bonferroni, ${ }^{* * *} p<.001$ ). Bar diagram in the right panel shows the mean EPSP amplitude as \% of control $30 \mathrm{~min}$ after HFS for both cell populations (LTP M4-EGFP MSNs $=159.2 \% \pm 12.9 \%$ vs. No LTP M4-EGFP MSNs $=85.29 \% \pm$ $15.5 \%$, Student $t$ test, ${ }^{* * *} p<.001$ ). (D) Left panel shows the application of $5 \mu \mathrm{mol} / \mathrm{L}$ U0126 prevented LTP induction in roughly the same percentage of MSNs recorded from dyskinetic mice expressing EGFP in iMSNs (A2A-EGFP) (LTP A2A-EGFP MSNs, $n=3$ vs. No LTP A2A-EGFP MSNs, $n=3$, two-way analysis of variance, $p<.0001$, post hoc Bonferroni, ${ }^{* * *} p<.001$ ). Histogram in the right panel shows the mean EPSP amplitude as \% of control 30 min after HFS for both cell populations (LTP A2A-EGFP MSNs $=129.7 \% \pm 2.4 \%$ vs. No LTP A2A-EGFP MSNs $=84.4 \% \pm 2.2 \%$, Student $t$ test, ${ }^{* * *} p<.001$ ). CTRL, control; dMSN, direct pathway MSN; EGFP, enhanced green fluorescent protein; EPSP, excitatory postsynaptic potential; HFS, high-frequency stimulation; LTP, long-term potentiation; MSN, medium spiny neuron; WT, wild-type.

One possibility is that Ras-GRF1 loss in the indirect pathway is either compensated by other Ras-specific exchange factors (e.g., Ras-GRF2 or CaIDGEFI/II) or that DARPP-32 activation is sufficient to increase ERK activity by acting downstream in the pathway, effectively bypassing Ras requirement. We also provided biochemical evidence supporting the idea that ERK activation in response to HFS occurs in both striatal pathways, although RasGRF1 is necessary only in dMSNs. The fact that ERK signaling activity is stronger in the iMSNs may be due to the higher excitability of these neurons compared with the dMSNs, as previously shown $(24,32,33)$.

Third, we observed that ERK signaling is also necessary for the reversal of HFS-LTP (i.e., synaptic depotentiation), a still poorly understood phenomenon, often associated with LTD and the action of protein phosphatases $(7,34)$. Fourth, our data support the idea that depotentiation and HFS-LTD in the striatum are distinct processes because the latter does not require ERK signaling. To support this evidence, we have provided data both using a chemical inhibitor of ERK signaling and using Ras-GRF1 mutant mice. In both cases, HFS-LTD is spared, and depotentiation is blocked.

This study demonstrates that in DA-denervated and dyskinetic animals, corticostriatal plasticity dependent on L-DOPA is profoundly altered. First, although L-DOPA causes less LID in RasGRF1 mutants, it restores HFS-LTP in both direct pathway and indirect pathway MSNs. Because LTP in the direct pathway is 
absent in naïve Ras-GRF1 mutants, we suggest that a L-DOPAdependent mechanism occurs in this subset of MSNs after DA denervation that may bypass the Ras-GRF1 requirement. However, although this LTP phenomenon may be relevant for the restoration of motor functions in response to L-DOPA, it is unlikely to be associated with the changes in dyskinesia because depotentiation appears to be the most crucial synaptic process involved in LID (7).

Finally, another surprising aspect of dopaminergic transmission was revealed after denervation and induction of LID. In marked contrast to the naïve condition, depotentiation in both indirect pathway and direct pathway MSNs of L-DOPA-treated slices is facilitated in the absence of Ras-GRF1, despite the overall lower ERK activation found in these mice (22). The idea that ERK signaling in slices from dyskinetic animals occludes rather than permits depotentiation in most MSNs was independently confirmed by direct MAPK inhibition in WT dyskinetic slices treated with U0126. The contrast between MSNs from naïve mice and MSNs from L-DOPA-treated mice may be in the altered activity of cytoplasmic or nuclear targets that could affect ERK requirement. It is conceivable that in dyskinetic animals, the abnormal hyperactivation of ERK may saturate LTP and may also block its reversal. In any case, our data support the idea that synaptic depotentiation in naïve slices and synaptic depotentiation in L-DOPAtreated slices are distinct synaptic processes.

In conclusion, the data obtained from the L-DOPA-treated RasGRF1 KO slices could suggest that, in contrast to the naïve condition, ERK signaling is not required for this form of LTP. However, further investigation using the MEK inhibitor U0126 revealed a much more complex scenario. We found that ERK signaling is required to elicit LTP in dyskinetic mice, but only in $\sim 50 \%$ of the cells. Unexpectedly, this segregation is not pathway dependent because it occurs in an apparently stochastic manner in both striatonigral and striatopallidal MSNs. All-or-none synaptic plasticity may occur in the CA3-CA1 hippocampal pathway, in which it appears that the initial synaptic state controls the behavior of individual synapses $(35,36)$. At the signaling level, it has been suggested that the ERK cascade may be a bistable system governed by both positive and negative feedback loops $(37,38)$. In addition, ERK signaling may be subjected to oscillatory behavior, as observed in some cellular systems (39). Little is known about these ERK regulatory processes in the brain and specifically in the MSNs of the striatum. However, without providing direct data to support the hypothesis, we speculate that ERK oscillations may exist in the brain and that these oscillations may be enhanced in DA-depleted animals treated with pulsatile L-DOPA, either in amplitude or in frequency, also altering the intrinsic oscillatory behavior of MSNs $(40,41)$. The effect of HFS on synaptic activity in a DA-depleted condition may depend on the temporal integration between the administration of L-DOPA and the oscillatory state involving ERK signaling. Also, in patients treated with L-DOPA, the so-called wearing-off phenomenon has been described as highly associated with rapid changes in DA levels, although these changes may not be related to dyskinesia itself but rather to other aspects of the L-DOPA treatment (42-44). Whether alterations in the neurotransmitter availability in the striatum may also result in altered oscillations of ERK activity remains to be demonstrated, but it is an interesting possibility to be investigated in the future.

This paper is dedicated to the memory of Prof. Renata Zippel (1948-2011). We thank Paul Kemp for granting us laboratory space, Raffaella Tonini for critical reading the manuscript and Marianne Benoit-Marand for her initial crucial help with technical issues.
The work was supported by the Michael J. Fox Foundation for Parkinson's Research (to RB), by Parkinson's UK (to RB), the Italian Ministry of Health (to RB, $P C$, and BP), the Compagnia di San Paolo (to $R B$ and $P C$ ) and the European Community $(E C)$ contract number 222918 (REPLACES) FP7 Thematic priority HEALTH (to PC).

The authors report no biomedical financial interests or potential conflicts of interest.

Supplementary material cited in this article is available online at http://dx.doi.org/10.1016/j.biopsych.2014.04.002.

1. Calabresi P, Picconi B, Tozzi A, Di Filippo M (2007): Dopaminemediated regulation of corticostriatal synaptic plasticity. Trends Neurosci 30:211-219.

2. Kreitzer AC, Malenka RC (2008): Striatal plasticity and basal ganglia circuit function. Neuron 60:543-554.

3. Surmeier DJ, Plotkin J, Shen W (2009): Dopamine and synaptic plasticity in dorsal striatal circuits controlling action selection. Curr Opin Neurobiol 19:621-628.

4. Calabresi P, Maj R, Pisani A, Mercuri NB, Bernardi G (1992): Long-term synaptic depression in the striatum: Physiological and pharmacological characterization. J Neurosci 12:4224-4233.

5. Calabresi P, Pisani A, Mercuri NB, Bernardi G (1992): Long term potentiation in the striatum is unmasked by removing the voltagedependent blockade of NMDA receptor channel. Eur J Neurosci 4: 929-935.

6. Centonze D, Gubellini P, Picconi B, Calabresi P, Giacomini P, Bernardi G (1999): Unilateral dopamine denervation blocks corticostriatal LTP. J Neurophysiol 82:3575-3579.

7. Picconi B, Centonze D, Hakansson K, Bernardi G, Greengard P, Fisone G, et al. (2003): Loss of bidirectional striatal synaptic plasticity in L-DOPA-induced dyskinesia. Nat Neurosci 6:501-506.

8. Suarez LM, Solis O, Carames JM, Taravini IR, Solis JM, Murer MG, et al. (2013): L-DOPA treatment selectively restores spine density in dopamine receptor D2-expressing projection neurons in dyskinetic mice [published online ahead of print Jun 12]. Biol Psychiatry.

9. Picconi B, Pisani A, Barone I, Bonsi P, Centonze D, Bernardi G, et al. (2005): Pathological synaptic plasticity in the striatum: Implications for Parkinson's disease. Neurotoxicology 26:779-783.

10. Picconi B, Bagetta V, Ghiglieri V, Paille V, Di Filippo M, Pendolino V, et al. (2010): Inhibition of phosphodiesterases rescues striatal long-term depression and reduces levodopa-induced dyskinesia. Brain 134:375-387.

11. Thomas GM, Huganir RL (2004): MAPK cascade signalling and synaptic plasticity. Nat Rev 5:173-183.

12. Sweatt JD (2004): Mitogen-activated protein kinases in synaptic plasticity and memory. Curr Opin Neurobiol 14:311-317.

13. Davis S, Laroche S (2006): Mitogen-activated protein kinase/extracellular regulated kinase signalling and memory stabilization: A review. Genes Brain Behav 5(suppl 2):61-72.

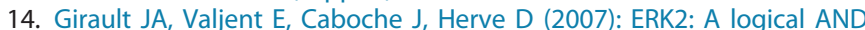
gate critical for drug-induced plasticity? Curr Opin Pharmacol 7:77-85.

15. Fasano S, Brambilla R (2011): Ras-ERK signaling in behavior: Old questions and new perspectives. Front Behav Neurosci 5:79.

16. Feyder M, Bonito-Oliva A, Fisone G (2011): L-DOPA-induced dyskinesia and abnormal signaling in striatal medium spiny neurons: Focus on dopamine D1 receptor-mediated transmission. Front Behav Neurosci 5:71.

17. Shiflett MW, Balleine BW (2010): Contributions of ERK signaling in the striatum to instrumental learning and performance. Behav Brain Res 218:240-247.

18. Calabresi $P$, Gubellini P, Picconi B, Centonze D, Pisani A, Bonsi $P$, et al. (2001): Inhibition of mitochondrial complex II induces a long-term potentiation of NMDA-mediated synaptic excitation in the striatum requiring endogenous dopamine. J Neurosci 21:5110-5120.

19. Xie GQ, Wang SJ, Li J, Cui SZ, Zhou R, Chen L, et al. (2009): Ethanol attenuates the HFS-induced, ERK-mediated LTP in a dose-dependent manner in rat striatum. Alcohol Clin Exp Res 33:121-128.

20. Brambilla R, Gnesutta N, Minichiello L, White G, Roylance AJ, Herron CE, et al. (1997): A role for the Ras signalling pathway in synaptic transmission and long-term memory. Nature 390:281-286. 
21. Fasano S, D'Antoni A, Orban PC, Valjent E, Putignano $E$, Vara $H$, et al. (2009): Ras-guanine nucleotide-releasing factor 1 (Ras-GRF1) controls activation of extracellular signal-regulated kinase (ERK) signaling in the striatum and long-term behavioral responses to cocaine. Biol Psychiatry 66:758-768.

22. Fasano S, Bezard E, D'Antoni A, Francardo V, Indrigo M, Qin L, et al. (2010): Inhibition of Ras-guanine nucleotide-releasing factor 1 (Ras-GRF1) signaling in the striatum reverts motor symptoms associated with L-dopa-induced dyskinesia. Proc Natl Acad Sci U S A 107:21824-21829.

23. Gong S, Zheng C, Doughty ML, Losos K, Didkovsky N, Schambra UB, et al. (2003): A gene expression atlas of the central nervous system based on bacterial artificial chromosomes. Nature 425:917-925.

24. Nazzaro C, Greco B, Cerovic M, Baxter P, Rubino T, Trusel M, et al. (2012): SK channel modulation rescues striatal plasticity and control over habit in cannabinoid tolerance. Nat Neurosci 15:284-293.

25. Orellana D, Morella IM, Indrigo M, Papale A, Brambilla R (2012): The ERK cascade in neuronal cell signaling. In: Mukai $\mathrm{H}$, editor. Protein Kinase Technologies. New York: Springer, 133-152.

26. Murer MG, Moratalla R (2011): Striatal signaling in L-DOPA-induced dyskinesia: Common mechanisms with drug abuse and long term memory involving D1 dopamine receptor stimulation. Front Neuroanat 5:51.

27. Calabresi P, Di Filippo M, Ghiglieri V, Picconi B (2008): Molecular mechanisms underlying levodopa-induced dyskinesia. Mov Disord 23(suppl 3):S570-S579.

28. Cenci MA (2007): Dopamine dysregulation of movement control in L-DOPA-induced dyskinesia. Trends Neurosci 30:236-243.

29. Jenner P (2008): Molecular mechanisms of L-DOPA-induced dyskinesia. Nat Rev 9:665-677.

30. Bertran-Gonzalez J, Bosch C, Maroteaux M, Matamales M, Herve D, Valjent E, et al. (2008): Opposing patterns of signaling activation in dopamine D1 and D2 receptor-expressing striatal neurons in response to cocaine and haloperidol. J Neurosci 28:5671-5685.

31. Santini E, Alcacer C, Cacciatore S, Heiman M, Herve D, Greengard P, et al. (2009): L-DOPA activates ERK signaling and phosphorylates histone $\mathrm{H} 3$ in the striatonigral medium spiny neurons of hemiparkinsonian mice. J Neurochem 108:621-633.
32. Kreitzer AC, Malenka RC (2007): Endocannabinoid-mediated rescue of striatal LTD and motor deficits in Parkinson's disease models. Nature 445:643-647.

33. Shen W, Flajolet M, Greengard P, Surmeier DJ (2008): Dichotomous dopaminergic control of striatal synaptic plasticity. Science (New York, NY) 321:848-851.

34. Malenka RC, Bear MF (2004): LTP and LTD: An embarrassment of riches. Neuron 44:5-21.

35. Petersen CC, Malenka RC, Nicoll RA, Hopfield JJ (1998): All-or-none potentiation at CA3-CA1 synapses. Proc Natl Acad Sci U S A 95:4732-4737.

36. O'Connor DH, Wittenberg GM, Wang SS (2005): Graded bidirectional synaptic plasticity is composed of switch-like unitary events. Proc Natl Acad Sci U S A 102:9679-9684.

37. Bhalla US, lyengar R (1999): Emergent properties of networks of biological signaling pathways. Science (New York, NY) 283:381-387.

38. Ferrell JE Jr (2002): Self-perpetuating states in signal transduction: Positive feedback, double-negative feedback and bistability. Curr Opin Cell Biol 14:140-148

39. Shankaran H, Wiley HS (2010): Oscillatory dynamics of the extracellular signal-regulated kinase pathway. Curr Opin Genet Dev 20:650-655.

40. Stern EA, Kincaid AE, Wilson CJ (1997): Spontaneous subthreshold membrane potential fluctuations and action potential variability of rat corticostriatal and striatal neurons in vivo. J Neurophysiol 77: $1697-1715$

41. Wilson CJ, Kawaguchi Y (1996): The origins of two-state spontaneous membrane potential fluctuations of neostriatal spiny neurons. J Neurosci 16:2397-2410.

42. de la Fuente-Fernandez R, Sossi V, Huang Z, Furtado S, Lu JQ, Calne $D B$, et al. (2004): Levodopa-induced changes in synaptic dopamine levels increase with progression of Parkinson's disease: Implications for dyskinesias. Brain 127:2747-2754.

43. Stocchi F, Jenner P, Obeso JA (2010): When do levodopa motor fluctuations first appear in Parkinson's disease? Eur Neurol 63:257-266.

44. Porras G, De Deurwaerdere P, Li Q, Marti M, Morgenstern R, Sohr R, et al. (2014): L-dopa-induced dyskinesia: Beyond an excessive dopamine tone in the striatum. Sci Rep 4:3730. 